\title{
Multiplicity results for the prescribed scalar curvature on low spheres
}

\author{
Mohamed Ben Ayed And Mohameden Ould Ahmedou
}

\begin{abstract}
In this paper, we consider the problem of multiplicity of conformal metrics of prescribed scalar curvature on standard spheres $\mathbb{S}^{3}, \mathbb{S}^{4}$. Under generic conditions we establish some Morse Inequalities at Infinity, which give a lower bound on the number of solutions to the above problem in terms of the total contribution of its critical points at Infinity to the difference of topology between the level sets of the associated Euler-Lagrange functional. As a by-product of our arguments we derive a new existence result on $\mathbb{S}^{4}$ through an Euler-Hopf type formula.
\end{abstract}

Mathematics Subject Classification (2000): 58E05 (primary); 35J65, 53C21, 35B40 (secondary).

\section{Introduction and main results}

The prescribed scalar curvature problem on closed riemannian manifolds amounts to find a positive smooth solution to the following equation:

$$
\text { (SC) }-c_{n} \Delta_{g} u+R_{g} u=K u^{(n+2) /(n-2)}, u>0 \text { in } M
$$

where $\Delta_{g}$ is the Laplace Beltrami operator, $R_{g}$ denotes the scalar curvature and $K: M \rightarrow \mathbb{R}$ is a given function on the manifold. To this problem many papers have been devoted (see $[2-4,6,8-10,12,14,15,18,19,21-25,27,28,30,31,36-38]$ and the references therein).

The special case where the manifold is a sphere endowed with its standard metric deserves particular attention. Indeed due to Kazdan-Warner obstructions, conditions have to be imposed on the function $K$ to insure the existence.

In this paper we revisit the case of 3 and 4-dimensional standard spheres in order to give, under generic conditions, some multiplicity results.

To state our results we set up following conditions and notation.

Received December 3, 2007; accepted in revised form May 27, 2008. 
Let for $n=3,4, K: \mathbb{S}^{n} \rightarrow \mathbb{R}$ be a $C^{2}$ positive function. We say that $K$ satisfies (H1) if for each critical point $y$ we have either

$$
y \text { is a nondegenerate critical point with } \Delta K(y) \neq 0
$$

or, there exist $\left(b_{i}\right)_{i \leq n} \subset \mathbb{R}^{*}$ such that in some geodesic normal coordinate centered at $y$ we have

$$
K(x)=K(y)+\sum_{i=1}^{n} b_{i}\left|(x-y)_{i}\right|^{\beta}+R(x-y) \quad \text { with } \quad b_{i} \neq 0 \text { and } \sum_{i=1}^{n} b_{i} \neq 0,
$$

where $\beta:=\beta(y) \in(2, n)$ and $\sum_{s=0}^{[\beta]}\left|\nabla^{s} R(y) \| y\right|^{-\beta-s}=o(1)$ as $y$ tends to zero. Here $\nabla^{s}$ denotes all possible derivatives of order $s$ and $[\beta]$ is the integer part of $\beta$. We denote by

$$
\begin{aligned}
& I_{1}:=\left\{y \in \mathbb{S}^{n}: \nabla K(y)=0 \text { and } \Delta K(y)<0\right\}, \\
& I_{2}:=\left\{y \in \mathbb{S}^{n}: \text { (1.2) is satisfied and } \sum_{i=1}^{n} b_{i}<0\right\} .
\end{aligned}
$$

On $\mathbb{S}^{4}$ we associate to each $p$-tuple of critical points of $K,\left(y_{i_{1}}, \cdots, y_{i_{p}}\right)$ with $y_{i_{l}} \in I_{1}$ for $l=1, \cdots, p$, a matrix $M\left(y_{i_{1}}, \ldots, y_{i_{p}}\right)=\left(m_{j q}\right)$ defined by

$$
m_{j j}=-\frac{\Delta K\left(y_{i_{j}}\right)}{3 K\left(y_{i_{j}}\right)^{2}}, \quad m_{j q}=-2 \frac{G\left(y_{i_{j}}, y_{i_{q}}\right)}{\left(K\left(y_{i_{j}}\right) K\left(y_{i_{q}}\right)\right)^{1 / 2}} \text { for } j \neq q,
$$

where $G$ is the Green's function of the conformal Laplacian $L_{g}:=-c_{n} \Delta_{g}+R_{g}$.

We say that $K$ satisfies (H2) if for each $p$-tuple of points $\left(y_{i_{1}}, \cdots, y_{i_{p}}\right)$ with $y_{i_{l}} \in I_{1}$ for $l=1, \cdots, p$ the corresponding matrix $M\left(y_{i_{1}}, \ldots, y_{i_{p}}\right)$ is nondegenerate. PDE

Now the problem that we are interested in, amount to solving the following

$$
\left(P_{K}\right) \quad-c_{n} \Delta_{g} u+R_{g} u=K u^{(n+2) /(n-2)}, u>0 \text { in } \mathbb{S}^{n} .
$$

We recall that a solution $u$ of $\left(P_{K}\right)$ is said to be nondegenerate if the linearized operator $\mathcal{L}(\varphi):=L_{g}(\varphi)-\frac{n+2}{n-2} u^{\frac{4}{n-2}} \varphi$ does not have zero as an eigenvalue.

Now we are ready to state our first multiplicity results.

Theorem 1.1. On $\mathbb{S}^{3}$ we assume that the function $K$ satisfies $(\mathrm{H} 1)$ and all solutions to the problem $\left(P_{K}\right)$ having their Morse indices less or equal than 3 are nondegenerate and denote their number by $N_{3}$. Then, we have

$$
\left|1-\sum_{y \in I_{1}}(-1)^{3-\imath(y)}-\sum_{y \in I_{2}}(-1)^{3-m(y)}\right| \leq N_{3},
$$

where, for $y \in I_{1}, \iota(y)$ denotes the Morse index of $K$ at $y$ and, for $y \in I_{2}, m(y):=$ $\#\left\{b_{i}: b_{i}<0\right\}$. 
Remark 1.2. We point out that from the Sard-Smale theorem it follows, that for generic $K$, the set of solutions of $\left(P_{K}\right)$ are all nondegenerate, see [37].

To state our next result we set $\mathcal{K}$ to be the set of all critical points of $K$ and

$$
\mathcal{K}^{+}:=\cup_{N}\left\{\left(q_{1}, \cdots, q_{N}\right) \in \mathcal{K}^{N} \text { such that } \varrho\left(q_{1}, \cdots, q_{N}\right)>0\right\}
$$

where $\varrho\left(q_{1}, \cdots, q_{N}\right)$ denotes the least eigenvalue of $M\left(q_{1}, \cdots, q_{N}\right)$.

Let $l^{+}:=\sup \left\{N ;\left(q_{1}, \cdots, q_{N}\right) \in \mathcal{K}^{+}\right\}$and $L_{0}:=\sup \left\{5 N-1-\sum_{j=1}^{N} \iota\left(q_{j}\right)\right.$ : $\left.\left(q_{1}, \cdots, q_{N}\right) \in \mathcal{K}^{+}\right\}$, where $\iota(y)$ denotes the Morse index of $K$ at the critical point $y$.

Theorem 1.3. On $\mathbb{S}^{4}$ we assume that $K$ satisfies $(\mathrm{H} 1)$ and $(\mathrm{H} 2)$ and all solutions to the problem $\left(P_{K}\right)$ having their Morse indices less or equal than $L_{0}$ are nondegenerate and denote their number by $N_{4}$. Then, we have

$$
\left|1-\sum_{y \in I_{2}}(-1)^{4-m(y)}-\sum_{s=1}^{l^{+}} \sum_{\varrho\left(y_{i_{1}}, \ldots, y_{i_{s}}\right)>0}(-1)^{5 s-1-\sum_{j=1}^{s} \iota\left(y_{i_{j}}\right)}\right| \leq N_{4},
$$

where, for $y \in I_{1}, \iota(y)$ denotes the Morse index of $K$ at $y$ and, for $y \in I_{2}, m(y):=$ $\#\left\{b_{i}: b_{i}<0\right\}$ and $\varrho$ denotes the least eigenvalue of the matrix $M$ defined in (1.3).

Observe that Theorems 1.1 and 1.3 are some sort of Morse type inequalities at Infinity in the sense that we give here a lower bound for the number of solutions in terms of the topology at infinity that is the total contribution of noncompact orbits of the gradient flow associated to the Euler Lagrange functional (its critical points at infinity). Recall that Morse inequalities give a lower bound on the number of critical points of a Morse function in terms of the Betti numbers of the underlying manifold. In our case the space of variation is contractible and hence has no topology. However due to the noncompactness of the problem there are critical points at infinity whose topological contribution to the difference of topology between the level sets of the functional can be computed thanks to a Morse lemma at Infinity which provides new coordinates in which the gradient flow takes a quite simple normal form.

In the following we give a brief description of the main ingredients behind the proof of Theorems 1.1 and 1.3.

Our argument uses a careful analysis of the lack of compactness of the Euler Lagrange functional $J$ (defined by (2.1) below) associated to problem $\left(P_{K}\right)$. Namely we study the noncompact orbits of the gradient flow of $J$ the so called critical points at infinity following the terminology of A. Bahri [9]. They are the noncompact orbits of $J$ along which $J$ is bounded and its gradient goes to zero. These critical points at infinity can be treated as usual critical points once a Morse Lemma at infinity is performed, from which we can derive just as in the classical Morse Theory the difference of topology induced by these noncompact orbits and compute their Morse indices. Such a Morse Lemma at infinity, which is a 
cornerstone in our analysis, is obtained through the construction of a suitable pseudogradient for which the Palais-Smale condition is satisfied along the decreasing flow lines, as long as these flow lines do not enter the neighborhood of a finite numbers of critical points of $K$ such that the related matrix $M$ (see (1.3)) is positive definite. Moreover along the flow lines of such a pseudogradient there can be only finitely many blow up points. Furthermore if some blow up points are close and the interactions between them is large, then the flow lines starting from there will exit the set $V(p, \varepsilon)$ and the functional $J$ will decrease with a fixed constant rate which depends only on $\varepsilon$.

A similar Morse Lemma has been established for the prescribed scalar curvature problem on the spheres $\mathbb{S}^{3}, \mathbb{S}^{4}$ under the hypothesis that the problem has no solution by A. Bahri and J. M. Coron [12], see also [14]. Since our aim is to prove multiplicity rather than only existence we have to perform our Morse Lemma without such an assumption, a situation which creates a new difficulty, namely to deal with the possibility of existence of a new type critical point at infinity consisting of a sum of bubbles plus a solution of $\left(P_{K}\right)$. Performing a Morse Lemma and constructing a pseudogradient near an $\varepsilon$-neighborhood of such a potential critical point at infinity, we rule out such a possibility on $\mathbb{S}^{3}$ and $\mathbb{S}^{4}$. Finally we notice that a statement similar to Theorem 1.1 for the prescribed curvature problem on the 3-dimensional sphere has been obtained by R. Schoen and D. Zhang [37], and due to the fact that in their case they assume the nondegeneracy condition $\Delta K \neq 0$ at critical points of $K$ their formula looks simpler. Their proof which is drastically different from ours involves a refined analysis for blowing up subcritical approximations.

As a by-product of the proof of Theorem 1.3 we have the following existence result which is a natural generalization of previous existence results obtained by Y.Y. Li [31] and Ben Ayed, Chen, Chtioui and Hammami [14].

Theorem 1.4. On $\mathbb{S}^{4}$ we assume that $K$ satisfies $(\mathrm{H} 1)$ and $(\mathrm{H} 2)$. Then, the problem $\left(P_{K}\right)$ has a solution if

$$
\left|1-\sum_{y \in I_{2}}(-1)^{4-m(y)}-\sum_{s=1}^{l^{+}} \sum_{\varrho\left(y_{i_{1}}, \ldots, y_{i_{s}}\right)>0}(-1)^{5 s-1-\sum_{j=1}^{s} \iota\left(y_{i_{j}}\right)}\right| \neq 0,
$$

where, for $y \in I_{1}, \iota(y)$ denotes the Morse index of $K$ at $y$ and, for $y \in I_{2}, m(y):=$ $\#\left\{b_{i}: b_{i}<0\right\}$ and $\varrho$ denotes the least eigenvalue of the matrix $M$ defined in (1.3).

ACKNOWLEDGEMENTS. Part of this work has been written when the authors enjoyed the hospitality of Centro di Ricerca Matematica Ennio De Giorgi in the framework of a research in pairs stay. They would like, in particular, to acknowledge the excellent working conditions.

This research was partly supported by the "Research in Pairs"-program of "Centro di Ricerca Matematica Ennio de Giorgi”. 


\section{Variational structure and preliminaries}

In this section we recall the functional setting, its variational structure and its main features. Problem $\left(P_{K}\right)$ has a variational structure. The Euler-Lagrange functional is

$$
J(u)=\frac{\|u\|^{2}}{\left(\int_{\mathbb{S}^{n}} K|u|^{2 n /(n-2)}\right)^{(n-2) / n}}
$$

defined on $H^{1}\left(\mathbb{S}^{n}, \mathbb{R}\right) \backslash\{0\}$ equipped with the norm

$$
\|u\|^{2}=\int_{\mathbb{S}^{n}}|\nabla u|^{2}+\frac{n(n-2)}{4} \int_{\mathbb{S}^{n}} u^{2} .
$$

We denote by $\Sigma$ the unit sphere of $H^{1}\left(\mathbb{S}^{n}, \mathbb{R}\right)$ and we set $\Sigma^{+}=\{u \in \Sigma: u \geq 0\}$. The Palais-Smale condition fails to be satisfied for $J$ on $\Sigma^{+}$. In order to characterize the sequences failing the Palais-Smale condition, we need to introduce some notations.

For $a \in \mathbb{S}^{n}$ and $\lambda>0$, let

$$
\delta_{(a, \lambda)}(x)=c_{0} \frac{\lambda^{(n-2) / 2}}{\left(\lambda^{2}+1+\left(1-\lambda^{2}\right) \cos d(a, x)\right)^{(n-2) / 2}}
$$

where $d$ is the geodesic distance on $\left(\mathbb{S}^{n}, g\right)$ and $c_{0}$ is chosen so that

$$
-\Delta \delta_{(a, \lambda)}+(n(n-2) / 4) \delta_{(a, \lambda)}=\delta_{(a, \lambda)}^{(n+2) /(n-2)} \quad \text { in } \mathbb{S}^{n} .
$$

For $\varepsilon>0$ and $p \in \mathbb{N}^{*}$, let us define

$$
\begin{aligned}
& V(p, \varepsilon)=\left\{u \in \Sigma / \exists a_{i} \in \mathbb{S}^{n}, \lambda_{i}>\varepsilon^{-1}, \alpha_{i}>0 \text { for } i=1, \ldots, p\right. \text { such that } \\
& \left.\left\|u-\sum_{i=1}^{p} \alpha_{i} \delta_{i}\right\|<\varepsilon,\left|\frac{\alpha_{i}^{4 /(n-2)} K\left(a_{i}\right)}{\alpha_{j}^{4 /(n-2)} K\left(a_{j}\right)}-1\right|<\varepsilon, \text { and } \varepsilon_{i j}<\varepsilon\right\}
\end{aligned}
$$

where $\delta_{i}=\delta_{\left(a_{i}, \lambda_{i}\right)}$ and $\varepsilon_{i j}=\left(\lambda_{i} / \lambda_{j}+\lambda_{j} / \lambda_{i}+\lambda_{i} \lambda_{j}\left(1-\cos d\left(a_{i}, a_{j}\right)\right) / 2\right)^{\frac{2-n}{2}}$. For $w$ a solution of $\left(P_{K}\right)$ we also define $V(p, \varepsilon, w)$ as

$$
\left\{u \in \Sigma / \exists \alpha_{0}>0 \text { such that } u-\alpha_{0} w \in V(p, \varepsilon) \text { and }\left|\alpha_{0}^{\frac{4}{n-2}} J(u)^{\frac{n}{n-2}}-1\right|<\varepsilon\right\} .
$$

The failure of the Palais-Smale condition can be described as follows. 
Proposition 2.1. $[12,33,40]$ Let $\left(u_{j}\right) \in \Sigma^{+}$be a sequence such that $\nabla J\left(u_{j}\right)$ tends to zero and $J\left(u_{j}\right)$ is bounded. Then, there exist an integer $p \in \mathbb{N}^{*}$, a sequence $\varepsilon_{j}>0, \varepsilon_{j}$ tends to zero, and an extracted of $u_{j}$ 's, again denoted $u_{j}$, such that $u_{j} \in V\left(p, \varepsilon_{j}, w\right)$ where $w$ is zero or a solution of $\left(P_{K}\right)$.

We consider the following minimization problem for $u \in V(p, \varepsilon)$ with $\varepsilon$ small

$$
\min _{\alpha_{i}>0, \lambda_{i}>0, a_{i} \in \mathbb{S}^{n}}\left\|u-\sum_{i=1}^{p} \alpha_{i} \delta_{\left(a_{i}, \lambda_{i}\right)}\right\|
$$

We then have the following proposition which defines a parametrization of the set $V(p, \varepsilon)$.

Proposition 2.2. $[9,12,35]$ For any $p \in \mathbb{N}^{*}$, there is $\varepsilon_{p}>0$ such that if $\varepsilon<\varepsilon_{p}$ and $u \in V(p, \varepsilon)$, the minimization problem (2.3) has a unique solution (up to permutation). In particular, we can write $u \in V(p, \varepsilon)$ as follows

$$
u=\sum_{i=1}^{p} \bar{\alpha}_{i} \delta_{\left(\bar{a}_{i}, \bar{\lambda}_{i}\right)}+v
$$

where $\left(\bar{\alpha}_{1}, \ldots, \bar{\alpha}_{p}, \bar{a}_{1}, \ldots, \bar{a}_{p}, \bar{\lambda}_{1}, \ldots, \bar{\lambda}_{p}\right)$ is the solution of $(2.3)$ and $v \in H^{1}\left(\mathbb{S}^{n}\right)$ such that

$$
\left(V_{0}\right) \quad\|v\| \leq \varepsilon, \quad(v, \psi)=0 \text { for } \psi \in \bigcup_{i \leq p, j \leq n}\left\{\delta_{i}, \frac{\partial \delta_{i}}{\partial \lambda_{i}}, \frac{\partial \delta_{i}}{\partial\left(a_{i}\right)^{j}}\right\},
$$

where $\left(a_{i}\right)^{j}$ denotes the $j^{\text {th }}$ component of $a_{i}$ and (., .) is the inner scalar associated to the norm $\|$.$\| .$

In the following we will say that $v \in\left(V_{0}\right)$ if $v$ satisfies $\left(V_{0}\right)$.

Proposition 2.3. [9,35] There exists a $C^{1}$ map which, to each

$$
\left(\alpha_{1}, \ldots, \alpha_{p}, a_{1}, \ldots, a_{p}, \lambda_{1}, \ldots, \lambda_{p}\right)
$$

such that $\sum_{i=1}^{p} \alpha_{i} \delta_{\left(a_{i}, \lambda_{i}\right)} \in V(p, \varepsilon)$ with small $\varepsilon$, associates $\bar{v}=\bar{v}_{\left(\alpha_{i}, a_{i}, \lambda_{i}\right)}$ satisfying

$$
J\left(\sum_{i=1}^{p} \alpha_{i} \delta_{\left(a_{i}, \lambda_{i}\right)}+\bar{v}\right)=\min _{v \in\left(V_{0}\right)} J\left(\sum_{i=1}^{p} \alpha_{i} \delta_{\left(a_{i}, \lambda_{i}\right)}+v\right) .
$$


Moreover, there exists $c>0$ such that the following holds

$$
\|\bar{v}\| \leq c\left(\sum_{i \leq p}\left(\frac{\left|\nabla K\left(a_{i}\right)\right|}{\lambda_{i}}+\frac{1}{\lambda_{i}^{2}}\right)+\sum_{k \neq r} \varepsilon_{k r}\left(\log \left(\varepsilon_{k r}^{-1}\right)\right)^{(n-2) / n}\right) .
$$

Let $w$ be a nondegenerate solution of $\left(P_{K}\right)$. The following proposition defines a parameterization of the set $V(p, \varepsilon, w)$.

Proposition 2.4. [10] There is $\varepsilon_{0}>0$ such that if $\varepsilon \leq \varepsilon_{0}$ and $u \in V(p, \varepsilon, w)$, then the problem

$$
\min _{\alpha_{i}>0, \lambda_{i}>0, a_{i} \in \mathbb{S}^{n}, h \in T_{w}\left(W_{u}(w)\right)}\left\|u-\sum_{i=1}^{p} \alpha_{i} \delta_{\left(a_{i}, \lambda_{i}\right)}-\alpha_{0}(w+h)\right\|
$$

has a unique solution $(\bar{\alpha}, \bar{\lambda}, \bar{a}, \bar{h})$. Thus, we write $u$ as follows:

$$
u=\sum_{i=1}^{p} \bar{\alpha}_{i} \delta_{\left(\bar{a}_{i}, \bar{\lambda}_{i}\right)}+\bar{\alpha}_{0}(w+\bar{h})+v
$$

where $v$ belongs to $H^{1}\left(\mathbb{S}^{n}\right) \cap T_{w}\left(W_{s}(w)\right)$ and it satisfies $\left(V_{0}\right), T_{w}\left(W_{u}(w)\right)$ and $T_{w}\left(W_{s}(w)\right)$ are the tangent spaces at $w$ to the unstable and stable manifolds of $w$.

Following A. Bahri we set the following definitions and notations.

Definition 2.5. A critical point at infinity of $J$ on $\Sigma^{+}$is a limit of a flow line $u(s)$ of the equation:

$$
\left\{\begin{array}{l}
\frac{\partial u}{\partial s}=-\nabla J(u) \\
u(0)=u_{0}
\end{array}\right.
$$

such that $u(s)$ remains in $V(p, \varepsilon(s), w)$ for $s \geq s_{0}$.

Here $w$ is either zero or a solution of $\left(P_{K}\right)$ and $\varepsilon(s)$ is some function tending to zero when $s \rightarrow \infty$. Using Proposition 2.4, $u(s)$ can be written as:

$$
u(s)=\sum_{i=1}^{p} \alpha_{i}(s) \delta_{\left(a_{i}(s), \lambda_{i}(s)\right)}+\alpha_{0}(s)(w+h(s))+v(s) .
$$

Denoting $a_{i}:=\lim _{s \rightarrow \infty} a_{i}(s)$ and $\alpha_{i}=\lim _{s \rightarrow \infty} \alpha_{i}(s)$, we let

$$
\left(a_{1}, \cdots, a_{p}, w\right)_{\infty} \text { or } \sum_{i=1}^{p} \alpha_{i} \delta_{\left(a_{i}, \infty\right)}+\alpha_{0} w
$$

such a critical point at infinity. If $w \neq 0$ it is called of $w$-type. 
With such a critical point at infinity are associated stable and unstable manifolds. These manifolds can be easily described once a Morse type reduction is performed, see $[10,14]$.

Definition 2.6. $z_{\infty}$ is said to be dominated by another critical point at infinity $z_{\infty}^{\prime}$ if

$$
W_{u}\left(z_{\infty}^{\prime}\right) \cap W_{s}\left(z_{\infty}\right) \neq \emptyset
$$

If we assume that the intersection is transverse, then we obtain

$$
\text { index }\left(z_{\infty}^{\prime}\right) \geq \operatorname{index}\left(z_{\infty}\right)+1
$$

\section{Characterization of the critical points at infinity}

This section is devoted to characterize the critical points at infinity. To this aim, we need to expand the functional in the potential sets $V(p, \varepsilon, w)$ where $w$ is a critical point of $J$ or zero and $p \in \mathbb{N}^{*}$. Moreover, we need to construct some pseudogradients on these sets which satisfy the Palais-Smale condition far away the critical points at infinity. First we will prove that in $V(p, \varepsilon, w)$, for $w \neq \equiv 0$, there is no critical points at infinity. Secondly, we prove that for $p \geq 2$, the sets $V(p, \varepsilon)$ do not contain critical point at infinity in the case of the three dimensional sphere. Then we prove Morse Lemmas at infinity in $V(1, \varepsilon)$ and $V(p, \varepsilon)$ with $p \geq 2$ for $n=4$.

\subsection{Ruling out the existence of critical point at Infinity in $V(p, \varepsilon, w)$ for $w \neq 0$}

In this section, for $u \in V(p, \varepsilon, w)$, using Proposition 2.4, we will write $u=$ $\sum_{i=1}^{p} \alpha_{i} \delta_{\left(a_{i}, \lambda_{i}\right)}+\alpha_{0}(w+h)+v$.

Proposition 3.1. For $\varepsilon>0$ small enough and $u=\sum_{i=1}^{p} \alpha_{i} \delta_{\left(a_{i}, \lambda_{i}\right)}+\alpha_{0}(w+h)+$ $v \in V(p, \varepsilon, w)$, we have the following expansion

$$
\begin{aligned}
J(u)= & \frac{S_{n} \sum_{i=1}^{p} \alpha_{i}^{2}+\alpha_{0}^{2}\|w\|^{2}}{\left(S_{n} \sum_{i=1}^{p} \alpha_{i}^{\frac{2 n}{n-2}} K\left(a_{i}\right)+\alpha_{0}^{\frac{2 n}{n-2}}\|w\|^{2}\right)^{\frac{n-2}{n}}}\left[1-c_{2} \alpha_{0} \sum_{i=1}^{p} \alpha_{i} \frac{w\left(a_{i}\right)}{\lambda_{i}^{(n-2) / 2}}\right. \\
& -c_{2} \sum_{i \neq j} \alpha_{i} \alpha_{j} \varepsilon_{i j}+f_{1}(v)+Q_{1}(v, v)+f_{2}(h)+\alpha_{0}^{2} Q_{2}(h, h) \\
& \left.+o\left(\sum_{i \neq j} \varepsilon_{i j}+\sum_{i=1}^{p} \frac{1}{\lambda_{i}^{(n-2) / 2}}+\|v\|^{2}+\|h\|^{2}\right)\right]
\end{aligned}
$$


where

$$
\begin{aligned}
Q_{1}(v, v) & =\frac{1}{\gamma_{1}}\|v\|^{2}-\frac{n+2}{\beta_{1}(n-2)} \int_{\mathbb{S}^{n}} K\left(\sum_{i=1}^{p}\left(\alpha_{i} \delta_{i}\right)^{\frac{4}{n-2}}+\left(\alpha_{0} w\right)^{\frac{4}{n-2}}\right) v^{2}, \\
Q_{2}(h, h) & =\frac{1}{\gamma_{1}}\|h\|^{2}-\frac{n+2}{\beta_{1}(n-2)} \int_{\mathbb{S}^{n}} K\left(\alpha_{0} w\right)^{\frac{4}{n-2}} h^{2}, \\
f_{1}(v) & =-\frac{1}{\beta_{1}} \int_{\mathbb{S}^{n}} K\left(\sum_{i=1}^{p} \alpha_{i} \delta_{i}\right)^{\frac{n+2}{n-2}} v, \\
f_{2}(h) & =\frac{\alpha_{0}}{\gamma_{1}} \sum_{i} \alpha_{i}\left(\delta_{i}, h\right)-\frac{\alpha_{0}}{\beta_{1}} \int_{\mathbb{S}^{n}} K\left(\sum_{i} \alpha_{i} \delta_{i}+\alpha_{0} w\right)^{\frac{n+2}{n-2}} h, \\
c_{2} & =c_{0}^{\frac{2 n}{n-2}} \int_{\mathbb{R}^{n}} \frac{d x}{\left(1+|x|^{2}\right)^{\frac{n+2}{2}}}, \quad S_{n}=c_{0}^{\frac{2 n}{n-2}} \int_{\mathbb{R}^{n}} \frac{d x}{\left(1+|x|^{2}\right)^{n}} \\
\beta_{1} & =S_{n}\left(\sum_{i=1}^{p} \alpha_{i}^{\frac{2 n}{n-2}} K\left(a_{i}\right)\right)+\alpha_{0}^{\frac{2 n}{n-2}}\|w\|^{2}, \quad \gamma_{1}=S_{n}\left(\sum_{i=1}^{p} \alpha_{i}^{2}\right)+\alpha_{0}^{2}\|w\|^{2} .
\end{aligned}
$$

Proof. Before starting the proof, we mention that it will be convenient to perform some stereographic projection in order to reduce our problem to $\mathbb{R}^{n}$. Let $D^{1,2}\left(\mathbb{R}^{n}\right)$ denote the completion of $C_{c}^{\infty}\left(\mathbb{R}^{n}\right)$ with respect to Dirichlet norm. The stereographic projection $\pi_{a}$ through a point $a \in \mathbb{S}^{n}$ induces an isometry $i: H^{1}\left(\mathbb{S}^{n}\right) \rightarrow$ $D^{1,2}\left(\mathbb{R}^{n}\right)$ according to the following formula

$$
(i v)(x)=\left(\frac{2}{1+|x|^{2}}\right)^{\frac{n-2}{2}} v\left(\pi_{a}^{-1}(x)\right), \quad v \in H^{1}\left(\mathbb{S}^{n}\right), x \in \mathbb{R}^{n}
$$

In particular, one can check that the following holds true, for every $v \in H^{1}\left(\mathbb{S}^{n}\right)$

$$
\int_{\mathbb{S}^{n}}\left(|\nabla v|^{2}+\frac{n(n-2)}{4} v^{2}\right)=\int_{\mathbb{R}^{n}}|\nabla(i v)|^{2} \quad \text { and } \quad \int_{\mathbb{S}^{n}}|v|^{\frac{2 n}{n-2}}=\int_{\mathbb{R}^{n}}|i v|^{\frac{2 n}{n-2}} .
$$

In the sequel, we will identify the function $K$ and its composition with the stereographic projection $\pi_{a}$. We will also identify a point $b$ of $\mathbb{S}^{n}$ and its image by $\pi_{a}$. These facts will be assumed as understood in the sequel. We need to estimate

$$
N(u)=\|u\|^{2} \quad \text { and } \quad D^{\frac{n}{n-2}}=\int_{\mathbb{S}^{n}} K(x) u^{\frac{2 n}{n-2}} .
$$


Expanding $N(u)$, it is equal to

$\sum_{i=1}^{p} \alpha_{i}^{2}\left\|\delta_{i}\right\|^{2}+2 \alpha_{i} \alpha_{0}\left(\delta_{i}, w+h\right)+\alpha_{0}^{2}\left(\|h\|^{2}+\|w\|^{2}\right)+\|v\|^{2}+\sum_{i \neq j} \alpha_{i} \alpha_{j}\left(\delta_{i}, \delta_{j}\right)$

Observe that (see [9])

$$
\begin{aligned}
\left\|\delta_{i}\right\|^{2} & =S_{n}, \\
\left(\delta_{i}, \delta_{j}\right) & =c_{2} \varepsilon_{i j}(1+o(1)), \quad \text { for } i \neq j, \\
\left(\delta_{i}, w\right) & =\int_{\mathbb{S}^{n}} w \delta_{i}^{(n+2) /(n-2)}=c_{2} \frac{w\left(a_{i}\right)}{\lambda_{i}^{(n-2) / 2}}+o\left(\frac{1}{\lambda_{i}^{(n-2) / 2}}\right) .
\end{aligned}
$$

Thus

$$
\begin{aligned}
N= & \gamma_{1}+2 \alpha_{0} \sum_{i=1}^{p} c_{2} \alpha_{i} \frac{w\left(a_{i}\right)}{\lambda_{i}^{(n-2) / 2}}+\alpha_{i}\left(\delta_{i}, h\right)+c_{2} \sum_{i \neq j} \alpha_{i} \alpha_{j} \varepsilon_{i j} \\
& +\alpha_{0}^{2}\|h\|^{2}+\|v\|^{2}+o\left(\sum_{i=1}^{p} \frac{1}{\lambda_{i}^{(n-2) / 2}}+\sum_{i \neq j} \varepsilon_{i j}\right) .
\end{aligned}
$$

For the denominator, we write

$$
\begin{aligned}
D^{\frac{n}{n-2}}= & \int K\left(\sum_{i=1}^{p} \alpha_{i} \delta_{i}\right)^{\frac{2 n}{n-2}}+\int K\left(\alpha_{0} w\right)^{\frac{2 n}{n-2}} \\
& +\frac{2 n \alpha_{0}}{n-2} \int K\left(\sum_{i=1}^{p} \alpha_{i} \delta_{i}\right)^{\frac{n+2}{n-2}} w+\frac{2 n}{n-2} \alpha_{0}^{\frac{n+2}{n-2}} \int K\left(\sum_{i=1}^{p} \alpha_{i} \delta_{i}\right) w^{\frac{n+2}{n-2}} \\
& +\frac{2 n}{n-2} \int K\left(\sum_{i=1}^{p} \alpha_{i} \delta_{i}+\alpha_{0} w\right)^{\frac{n+2}{n-2}}\left(\alpha_{0} h+v\right) \\
& +\frac{n(n+2)}{(n-2)^{2}} \int K\left(\sum_{i=1}^{p} \alpha_{i} \delta_{i}+\alpha_{0} w\right)^{\frac{4}{n-2}}\left(\alpha_{0}^{2} h^{2}+v^{2}+2 \alpha_{0} h v\right) \\
& +O\left(\sum \int w^{\frac{4}{n-2}} \delta_{i}^{2}+w^{2} \delta_{i}^{\frac{4}{n-2}}\right)+O\left(\|v\|^{3}+\|h\|^{3}\right) .
\end{aligned}
$$


Observe that

$$
\begin{aligned}
& \int_{\mathbb{S}^{n}} K\left(\sum_{i=1}^{p} \alpha_{i} \delta_{i}\right)^{\frac{2 n}{n-2}}=\sum_{i=1}^{p} \alpha_{i}^{\frac{2 n}{n-2}} K\left(a_{i}\right) S_{n} \\
& +\frac{2 n}{n-2} c_{2} \sum_{i \neq j} \alpha_{i}^{\frac{n+2}{n-2}} \alpha_{j} K\left(a_{i}\right) \varepsilon_{i j}+O\left(\frac{1}{\lambda_{i}^{2}}\right)+o\left(\varepsilon_{i j}\right), \\
& \int_{\mathbb{S}^{n}} K w^{\frac{2 n}{n-2}}=\|w\|^{2} ; \quad \int_{\mathbb{S}^{n}} K w^{\frac{n+2}{n-2}} \delta_{i}=c_{2} \frac{w\left(a_{i}\right)}{\lambda_{i}^{(n-2) / 2}}+o\left(\frac{1}{\lambda_{i}^{(n-2) / 2}}\right), \\
& \int_{\mathbb{S}^{n}} K\left(\sum \alpha_{i} \delta_{i}\right)^{\frac{n+2}{n-2}} w=c_{2} \sum \alpha_{i}^{\frac{n+2}{n-2}} K\left(a_{i}\right) \frac{w\left(a_{i}\right)}{\lambda_{i}^{(n-2) / 2}}+o\left(\frac{1}{\lambda_{i}^{(n-2) / 2}}\right), \\
& \int_{\mathbb{S}^{n}} \delta_{i}^{\frac{4}{n-2}} w^{2}+\delta_{i}^{2} w^{\frac{4}{n-2}}=o\left(\frac{1}{\lambda_{i}^{(n-2) / 2}}\right) \\
& \int_{\mathbb{S}^{n}} K\left(\sum \alpha_{i} \delta_{i}+\alpha_{0} w\right)^{\frac{4}{n-2}} v h=O\left(\int\left(\sum \delta_{i}^{\frac{4}{n-2}}+w^{\frac{6-n}{n-2}} \sum \delta_{i}\right)|v||h|\right) \\
& =O\left(\|v\|^{3}+\|h\|^{3}+1 / \lambda_{i}^{3(n-2) / 2}\right),
\end{aligned}
$$

where we have used that $v \in T_{w}\left(W_{s}(w)\right)$ and $h$ belongs to $T_{w}\left(W_{u}(w)\right)$ which is a finite dimensional space. Hence it implies that $\|h\|_{\infty} \leq c\|h\|$.

Concerning the linear form of $v$, since $v \in T_{w}\left(W_{s}(w)\right)$, it can be written as

$$
\begin{aligned}
& \int_{\mathbb{S}^{n}} K\left(\sum_{i=1}^{p} \alpha_{i} \delta_{i}+\alpha_{0} w\right)^{\frac{n+2}{n-2}} v \\
& =\int K\left(\sum_{i=1}^{p} \alpha_{i} \delta_{i}\right)^{\frac{n+2}{n-2}} v+O\left(\sum_{i=1}^{p} \int\left(\delta_{i}^{\frac{4}{n-2}} w+\delta_{i} w^{\frac{4}{n-2}}\right)|v|\right) \\
& =f_{1}(v)+O\left(\frac{\|v\|}{\lambda_{i}^{(n-2) / 2}}\right) .
\end{aligned}
$$


Finally, we have

$$
\begin{aligned}
\int K\left(\sum_{i=1}^{p} \alpha_{i} \delta_{i}+\alpha_{0} w\right)^{\frac{4}{n-2}} h^{2} & =\alpha_{0}^{\frac{4}{n-2}} \int K w^{\frac{4}{n-2}} h^{2}+o\left(\|h\|^{2}\right) \\
\int K\left(\sum_{i=1}^{p} \alpha_{i} \delta_{i}+\alpha_{0} w\right)^{\frac{4}{n-2}} v^{2}= & \sum_{i=1}^{p} \int K\left(\alpha_{i} \delta_{i}\right)^{\frac{4}{n-2}} v^{2} \\
& +\alpha_{0}^{\frac{4}{n-2}} \int K w^{\frac{4}{n-2}} v^{2}+o\left(\|v\|^{2}\right) .
\end{aligned}
$$

Combining (3.4),..,(3.13), the result follows.

Now, we introduce the following lemma which is proved for the dimensions $n \geq 7$ in [10] and the proof still works for the lower dimensions.

Lemma 3.2. We have

(a) $Q_{1}(v, v)$ is a quadratic form positive definite in

$$
E_{v}=\left\{v \in H^{1}\left(\mathbb{S}^{n}\right) / v \in T_{w}\left(W_{s}(w)\right) \text { and } v \text { satisfies }\left(V_{0}\right)\right\}
$$

(b) $Q_{2}(h, h)$ is a quadratic form negative definite in $T_{w}\left(W_{u}(w)\right)$.

Corollary 3.3. [10] Let $u=\sum_{i=1}^{p} \alpha_{i} \delta_{\left(a_{i}, \lambda_{i}\right)}+\alpha_{0}(w+h)+v \in V(p, \varepsilon, w)$. There is an optimal $(\bar{v}, \bar{h})$ and a change of variables $v-\bar{v} \rightarrow V$ and $h-\bar{h} \rightarrow H$ such that

$$
J(u)=J\left(\sum_{i=1}^{p} \alpha_{i} \delta_{\left(a_{i}, \lambda_{i}\right)}+\alpha_{0} w+\bar{h}+\bar{v}\right)+\|V\|^{2}-\|H\|^{2} .
$$

Furthermore we have the following estimates

$$
\begin{gathered}
\|\bar{h}\| \leq \sum_{i} \frac{c}{\lambda_{i}^{\frac{n-2}{2}}} \text { and }\|\bar{v}\| \leq c \sum_{i} \frac{\left|\nabla K\left(a_{i}\right)\right|}{\lambda_{i}}+\frac{c}{\lambda_{i}^{2}}+c \sum \varepsilon_{k r}\left(\log \varepsilon_{k r}^{-1}\right)^{\frac{n-2}{n}}, \\
\quad S_{n} \sum_{i=1}^{p} \alpha_{i}^{2}+\alpha_{0}^{2}\|w\|^{2} \\
\left.\quad-c_{n} \sum_{i=1}^{p} \alpha_{i}^{\frac{2 n}{n-2}} K\left(a_{i}\right)+\alpha_{0}^{\frac{2 n}{n-2}}\|w\|^{2}\right)^{\frac{n-2}{n}}
\end{gathered}
$$


Proof. The expansion of $J$ with respect to $h$ (respectively to $v$ ) is very close, up to a multiplicative constant, to $Q_{2}(h, h)+f_{2}(h)$ (respectively $\left.Q_{1}(v, v)+f_{1}(v)\right)$. Since $Q_{2}$ is negative definite (respectively $Q_{1}$ is positive definite), there is a unique maximum $\bar{h}$ in the space of $h$ 's (respectively a unique minimum $\bar{v}$ in the space of $v$ ). Furthermore, it is easy to derive $\|\bar{h}\| \leq c\left\|f_{2}\right\|$ and $\|\bar{v}\| \leq c\left\|f_{1}\right\|$. The estimate of $\bar{v}$ follows from Proposition 2.3. For the estimate of $\bar{h}$, we use the fact that for each $h \in T_{w}\left(W_{u}(w)\right)$ which is a finite dimensional space, we have $\|h\|_{\infty} \leq c\|h\|$. Therefore, we derive that $\left\|f_{2}\right\|=O\left(\sum \lambda_{i}^{(2-n) / 2}\right)$. Then our result follows.

Corollary 3.4. Let $K$ be a $C^{2}$ positive function and let $w$ be a nondegenerate critical point of $J$ in $\Sigma^{+}$. Then, for each $p \in \mathbb{N}^{*}$, there is no critical points or critical points at infinity in the set $V(p, \varepsilon, w)$, that means we can construct a pseudogradient of $J$ so that the Palais-Smale condition is satisfied along the decreasing flow lines.

The proof follows immediately from the above corollary and the fact that $w>0$ in $\mathbb{S}^{n}$.

\subsection{Ruling out the critical point at infinity in $V(p, \varepsilon)$ for $p \geq 2$ and $n=3$}

This fact has been proven by A. Bahri and J. M. Coron [12] assuming (1.1). We will basically repeat the same argument to show that it still works under the assumption (1.2) as well.

Proposition 3.5. Assume that $K$ satisfies $(\mathrm{H} 1)$ and let $\beta:=\max \{\beta(y): y$ satisfies (1.2)\}. For any $p \geq 2$, there exists a pseudogradient $W$ so that the following holds:

There is a positive constant $c$ independent of $u=\sum_{i \leq p} \alpha_{i} \delta_{\left(a_{i}, \lambda_{i}\right)} \in V(p, \varepsilon)$ such that

(i) $(-\nabla J(u), W) \geq c \sum_{i=1}^{p} \frac{\left|\nabla K\left(a_{i}\right)\right|}{\lambda_{i}}+\frac{c}{\lambda_{i}^{\beta}}+c \sum_{k \neq r} \varepsilon_{k r}$

(ii) $\left(-\nabla J(u+\bar{v}), W+\frac{\partial \bar{v}}{\partial\left(\alpha_{i}, a_{i}, \lambda_{i}\right)}(W)\right) \geq c \sum_{i=1}^{p} \frac{\left|\nabla K\left(a_{i}\right)\right|}{\lambda_{i}}+\frac{c}{\lambda_{i}^{\beta}}+c \sum_{k \neq r} \varepsilon_{k r}$

(iii) $|W|$ is bounded. Furthermore, the maximum of the $\lambda_{i}$ 's is a decreasing function along the flow lines generated by $W$.

Before giving the proof we will give an immediate consequence of this proposition.

Corollary 3.6. Let $n=3$ and assume that $K$ satisfies $(\mathrm{H} 1)$. Then for each $p \geq 2$, in $V(p, \varepsilon)$ there is no critical point or critical point at infinity. 
Proof of Proposition 3.5. Ordering the $\lambda_{i}$ 's : $\lambda_{i_{1}} \leq \ldots \leq \lambda_{i_{p}}$ and let us define

$$
\begin{aligned}
& W_{1}^{1}:=-\sum_{j=2}^{p} 2^{j} \alpha_{i_{j}} \lambda_{i_{j}} \frac{\partial \delta_{i_{j}}}{\partial \lambda_{i_{j}}}, \\
& W_{1}^{2}=: \sum_{i=1}^{p} \frac{1}{\lambda_{i}} \frac{\partial \delta_{i}}{\partial a_{i}} \cdot \frac{\nabla K\left(a_{i}\right)}{\left|\nabla K\left(a_{i}\right)\right|} \psi_{1}\left(\lambda_{i}\left|\nabla K\left(a_{i}\right)\right|\right),
\end{aligned}
$$

where $\psi_{1}$ is a cut off function defined by $\psi_{1}(t)=0$ if $t \leq C$ and $\psi_{1}(t)=1$ if $t \geq 2 C$, and $C$ is a large positive constant.

An easy computation shows that

$$
\begin{aligned}
& -2 \lambda_{i} \frac{\partial \varepsilon_{i j}}{\partial \lambda_{i}}-\lambda_{j} \frac{\partial \varepsilon_{i j}}{\partial \lambda_{j}} \geq c \varepsilon_{i j} \text { for } \lambda_{j} \leq \lambda_{i}, \\
& \frac{1}{\lambda_{i_{j}}^{2}}=o\left(\varepsilon_{i_{1} i_{j}}\right) \text { for each } j \geq 2 .
\end{aligned}
$$

Hence using Proposition 5.1 we derive that

$$
\begin{aligned}
& \left(-\nabla J(u), W_{1}^{1}\right) \geq c \sum_{k \neq r} \varepsilon_{k r}+O\left(\sum_{j \geq 2} \frac{1}{\lambda_{i_{j}}^{2}}\right) \geq c \sum_{k \neq r} \varepsilon_{k r}+c \sum_{j \geq 2} \frac{1}{\lambda_{i_{j}}^{2}}, \\
& \left(-\nabla J(u), W_{1}^{2}\right) \geq c \sum_{i \leq p} \psi_{1}\left(\lambda_{i}\left|\nabla K\left(a_{i}\right)\right|\right) \frac{\left|\nabla K\left(a_{i}\right)\right|}{\lambda_{i}}+O\left(\sum \varepsilon_{k r}\right) .
\end{aligned}
$$

Now, define $W_{1}=W_{1}^{1}+m_{1} W_{1}^{2}$ where $m_{1}$ is a small positive constant. By using (3.16) and (3.17) we derive that

$$
\begin{gathered}
\left(-\nabla J(u), W_{1}\right) \geq c \sum_{k \neq r} \varepsilon_{k r}+\sum_{j \geq 2} \frac{c}{\lambda_{i_{j}}^{2}}+c \sum_{i \neq i_{1}} \frac{\left|\nabla K\left(a_{i}\right)\right|}{\lambda_{i}} \\
+c \psi_{1}\left(\lambda_{i_{1}}\left|\nabla K\left(a_{i_{1}}\right)\right|\right) \frac{\left|\nabla K\left(a_{i_{1}}\right)\right|}{\lambda_{i_{1}}} .
\end{gathered}
$$

Hence the proof of the proposition follows in the case where $\lambda_{i_{1}}\left|\nabla K\left(a_{i_{1}}\right)\right| \geq 2 C$, where $C$ is a large positive constant or $\lambda_{i_{1}}$ and $\lambda_{i_{2}}$ are of the same order. In the other case, we need to add the variable $\lambda_{i_{1}}$ in the lower bound. Note that since $\lambda_{i_{1}}\left|\nabla K\left(a_{i_{1}}\right)\right| \leq 2 C$, the concentration point $a_{i_{1}}$ is close to a critical point $y$ of $K$. Recall that $K$ satisfies (H1) which means that $y$ has to verify (1.1) or (1.2). Hence we have two cases. 
Case 1. $a_{i_{1}}$ is close to $y$ which satisfies (1.1).

We introduce $W_{2}$ as

$$
W_{2}:=-\Delta K(y) \lambda_{i_{1}} \frac{\partial \delta_{i_{1}}}{\partial \lambda_{i_{1}}}
$$

and using Proposition 5.1 we derive that

$$
\left(-\nabla J(u), W_{2}\right) \geq c \frac{1}{\lambda_{i_{1}}^{2}}+O\left(\sum_{k \neq r} \varepsilon_{k r}\right) .
$$

Now, we define $W:=W_{1}+m_{2} W_{2}$ where $m_{2}$ is a small positive constant. Hence, by (3.18) and (3.20), the proof of claim (i) follows in this case.

Case 2. $a_{i_{1}}$ is close to $y$ which satisfies (1.2).

We define

$$
\begin{aligned}
W_{3}^{1} & =\sum_{i=1}^{n} \frac{1}{\lambda_{i_{1}}} \frac{\partial \delta_{i_{1}}}{\partial\left(a_{i_{1}}\right)_{i}} \int_{\mathbb{R}^{n}} b_{i} \frac{\left|x_{i}+\lambda_{i_{1}}\left(a_{i_{1}}\right)_{i}\right|^{\beta}}{\left(1+\lambda_{i_{1}}\left|\left(a_{i_{1}}\right)_{i}\right|\right)^{\beta-1}} \frac{x_{i}}{\left(1+|x|^{2}\right)^{n+1}} d x \\
W_{3}^{2} & =\left(-\sum_{i=1}^{n} b_{i}\right) \lambda_{i_{1}} \frac{\partial \delta_{i_{1}}}{\partial \lambda_{i_{1}}} \psi_{2}\left(\lambda_{i_{1}}\left|a_{i_{1}}\right|\right),
\end{aligned}
$$

where $n=3$ and $\psi_{2}$ is a cut off function defined by $\psi_{2}(t)=1$ if $t \leq \eta$ and $\psi_{2}(t)=0$ if $t \geq 2 \eta$, where $\eta$ is a small positive constant.

First we remark that the pseudogradient $W_{3}^{1}$ is bounded. In fact, the claim is trivial if $\lambda_{i_{1}}\left|a_{i_{1}}\right|$ is bounded. In the other case, let $i$ such that $\lambda_{i_{1}}\left|\left(a_{i_{1}}\right)_{i}\right| \geq c \lambda_{i_{1}}\left|a_{i_{1}}\right|$, we see that

$$
\begin{aligned}
\int_{\mathbb{R}^{n}} \frac{\left|x_{i}+\lambda_{i_{1}}\left(a_{i_{1}}\right)_{i}\right|^{\beta} x_{i}}{\left(1+|x|^{2}\right)^{n+1}} d x & =\left(\lambda_{i_{1}}\left|\left(a_{i_{1}}\right)_{i}\right|\right)^{\beta} \int_{\mathbb{R}^{n}}\left|1+\frac{x_{i}}{\lambda_{i_{1}}\left|\left(a_{i_{1}}\right)_{i}\right|}\right|^{\beta} \frac{x_{i}}{\left(1+|x|^{2}\right)^{n+1}} d x \\
& =c\left(\lambda_{i_{1}}\left|\left(a_{i_{1}}\right)_{i}\right|\right)^{\beta-1}(1+o(1)) .
\end{aligned}
$$

In this case, the vector field will be defined by $W_{3}:=W_{3}^{1}+W_{3}^{2}$. Using Proposition 5.1, we get

$$
\begin{aligned}
\left(-\nabla J(u), W_{3}\right) \geq & \frac{c}{\lambda_{i_{1}}^{\beta}} \sum_{i=1}^{n} b_{i}^{2}\left(\int_{\mathbb{R}^{n}} \frac{\left|x_{i}+\lambda_{i_{1}}\left(a_{i_{1}}\right)_{i}\right|^{\beta}}{\left(1+\lambda_{i_{1}}\left|\left(a_{i_{1}}\right)_{i}\right|\right)^{(\beta-1) / 2}} \frac{x_{i}}{\left(1+|x|^{2}\right)^{n+1}} d x\right)^{2} \\
& +\psi_{2}\left(\lambda_{i_{1}}\left|a_{i_{1}}\right|\right)\left(\sum_{i=1}^{n} b_{i}\right)^{2} \frac{c^{\prime}}{\lambda_{i_{1}}^{\beta}}+o\left(\frac{1}{\lambda_{i_{1}}^{\beta}}\right)+O\left(\sum \varepsilon_{k r}\right) .
\end{aligned}
$$

We claim that

$$
\left(-\nabla J(u), W_{3}\right) \geq \frac{c}{\lambda_{i_{1}}^{\beta}}+c \frac{\left|\nabla K\left(a_{i_{1}}\right)\right|}{\lambda_{i_{1}}}+O\left(\sum \varepsilon_{k r}\right) .
$$


Recall that $y$ satisfies (1.2). Thus near $y$ we have $|\nabla K(x)| \sim c \sum\left|b_{i}\right|\left|x_{i}\right|^{\beta-1}$. Observe that, if $\lambda_{i_{1}}\left|a_{i_{1}}\right|$ is large then using (3.23) we can make appear $1 / \lambda_{i_{1}}^{\beta}$ and $\left|\nabla K\left(a_{i_{1}}\right)\right| / \lambda_{i_{1}}$ in the lower bound of (3.24) and our claim follows in this case.

Now, if $\lambda_{i_{1}}\left|a_{i_{1}}\right| \leq \eta$ (that means it is small), where $\eta$ is defined in the definition of $\psi_{2}$, then we have $\psi_{2}\left(\lambda_{i_{1}}\left|a_{i_{1}}\right|\right)=1$ and $\left|\nabla K\left(a_{i_{1}}\right)\right|$ is small with respect to $\lambda_{i_{1}}^{1-\beta}$. Thus (3.25) holds in this case.

Finally, if $\lambda_{i_{1}}\left|a_{i_{1}}\right|$ is bounded below and above, by using Lemma 5.2, we have

$$
\sum_{i}\left(\int_{\mathbb{R}^{n}} \frac{b_{i}\left|x_{i}+\lambda_{i_{1}}\left(a_{i_{1}}\right)_{i}\right|^{\beta}}{\left(1+\lambda_{i_{1}}\left|\left(a_{i_{1}}\right)_{i}\right|\right)^{(\beta-1) / 2}} \frac{x_{i}}{\left(1+|x|^{2}\right)^{n+1}} d x\right)^{2} \geq c>0 .
$$

Thus our claim follows and therefore (3.25) is proved.

Now, define $W=W_{1}+m_{2} W_{3}$, where $m_{2}$ is a small positive constant, and using (3.18) and (3.25), claim (i) follows.

Regarding claim (ii), it follows from claim (i) and the estimates of $\|\bar{v}\|^{2}$ and $\| \nabla J(u+\bar{v}\|\| \bar{v} \|$ as in [10] and [14]. Finally, claim (iii) follows from the definition of $W$.

The proof of the proposition is thereby completed.

\subsection{Morse Lemma at infinity in $V(1, \varepsilon)$ for $n=3,4$}

In this section, we will characterize the critical points at infinity in $V(1, \varepsilon)$. Following [10] and [14], we need to construct a vector field in this set which satisfies some required properties.

Proposition 3.7. Let $n=3,4$. Assume that $K$ satisfies $(\mathrm{H} 1)$ and let $\beta:=\max \{\beta(y)$ : $y$ satisfies (1.2)\}. Then, there exists a pseudogradient $W$ so that the following holds:

There is a positive constant $c>0$ independent of $u=\alpha \delta_{(a, \lambda)} \in V(1, \varepsilon)$ such that

(i) $(-\nabla J(u), W) \geq c \frac{|\nabla K(a)|}{\lambda}+\frac{1}{\lambda^{\beta}}$

(ii) $\left(-\nabla J(u+\bar{v}), W+\frac{\partial \bar{v}}{\partial\left(\alpha_{i}, a_{i}, \lambda_{i}\right)}(W)\right) \geq c \frac{|\nabla K(a)|}{\lambda}+\frac{1}{\lambda^{\beta}}$

(iii) $|W|$ is bounded. Furthermore, $\lambda$ is an increasing function along the flow lines generated by $W$ only if $a$ is close to a critical point $y \in I_{1} \cup I_{2}$.

Proof. The construction depends on the variables $a$ and $\lambda$. We will divide the set $V(1, \varepsilon)$ into three subsets:

$$
\begin{aligned}
& F_{1}:=\left\{\alpha \delta_{(a, \lambda)}: \lambda|\nabla K(a)| \geq C\right\}, \\
& F_{2}:=\left\{\alpha \delta_{(a, \lambda)}: \lambda|\nabla K(a)| \leq 2 C \text { and } a \text { is close to } y \text { satisfying (1.1) }\right\}, \\
& F_{3}:=\left\{\alpha \delta_{(a, \lambda)}: \lambda|\nabla K(a)| \leq 2 C \text { and } a \text { is close to } y \text { satisfying (1.2) }\right\},
\end{aligned}
$$

where $C$ is a large positive constant. 
In $F_{1}$, we define

$$
W_{1}:=\frac{1}{\lambda} \frac{\partial \delta}{\partial a} \frac{\nabla K(a)}{|\nabla K(a)|}
$$

and using Proposition 5.1 we get

$$
\left(-\nabla J(u), W_{1}\right) \geq c \frac{|\nabla K(a)|}{\lambda}+O\left(\frac{1}{\lambda^{2}}\right) \geq c \frac{|\nabla K(a)|}{\lambda}+\frac{c}{\lambda^{2}} .
$$

In $F_{2}$, we use the pseudogradient $W_{2}$ defined by (3.19) and using Proposition 5.1, we get

$$
\left(-\nabla J(u), W_{2}\right) \geq c \frac{|\Delta K(a)|^{2}}{\lambda^{2}}+o\left(\frac{1}{\lambda^{2}}\right) \geq \frac{c}{\lambda^{2}}+c \frac{|\nabla K(a)|}{\lambda} .
$$

It remains to define the pseudogradient in $F_{3}$. In this region we set $W_{3}=W_{3}^{1}+W_{3}^{2}$ where $W_{3}^{1}$ and $W_{3}^{2}$ are defined by (3.21) and (3.22) respectively. Now, following the proof of (3.25), we obtain

$$
\left(-\nabla J(u), W_{3}\right) \geq \frac{c}{\lambda^{\beta}}+c \frac{|\nabla K(a)|}{\lambda} .
$$

The required pseudogradient $W$ will be defined by convex combination of $W_{1}, W_{2}$ and $W_{3}$. Using (3.26), (3.27) and (3.28), claim (i) follows. Regarding claim (ii), it follows from claim (i) and the estimate of $\|\bar{v}\|^{2}$. Concerning claim (iii), it follows from the definition of $W$. Hence the proof of the proposition is completed.

Once the pseudogradient is constructed, following [10] and [14], we can find a change of variables which gives the normal form of the functional $J$ on the subset $F_{y}:=\left\{\alpha \delta_{(a, \lambda)}+v: a\right.$ is close to $\left.y\right\}$, where $y \in I_{1} \cup I_{2}$. More precisely, we have

Proposition 3.8. Let $n=3,4$ and assume that $K$ satisfies (H1). For $y \in I_{1} \cup I_{2}$, in $F_{y}:=\left\{\alpha \delta_{(a, \lambda)}+v:\right.$ a is close to $\left.y\right\}$, there exists a change of variables:

$$
v-\bar{v} \mapsto V \quad \text { and } \quad(a, \lambda) \mapsto(\tilde{a}, \tilde{\lambda})
$$

so that

$$
J\left(\alpha \delta_{(a, \lambda)}+v\right)=\frac{S_{n}^{2 / n}}{K(\tilde{a})^{(n-2) / n}}\left(1+c(1-\eta) \frac{g(y)}{\tilde{\lambda} \gamma(y)}\right)+\|V\|^{2},
$$

where $\eta$ is a small positive constant, $g(y)=-\Delta K(y)$ and $\gamma(y)=2$ if $y$ satisfies (1.1) and $g(y)=-\sum b_{i}$ and $\gamma(y)=\beta(y)$ if $y$ satisfies (1.2).

Proof. The proof is exactly the same as in [10] and [14]. So we omit it.

As a consequence of the above proposition, we have the following result: 
Corollary 3.9. Let $K$ be a positive function satisfying $(\mathrm{H} 1)$. For $n=3$, 4 , the only critical points at infinity in $V(1, \varepsilon)$ are $(y)_{\infty}$ where $y \in I_{1} \cup I_{2}$. Such a critical point has a Morse index equal to $n-i n d e x(K, y)$ where index $(K, y)$ is the Morse index of $K$ in case $y \in I_{1}$ and index $(K, y)=\#\left\{b_{i} ; b_{i}<0\right\}$ in case $y \in I_{2}$.

Proof. The claim follows from Propositions 3.7 and 3.8.

\subsection{Morse Lemma at infinity in $V(p, \varepsilon)$ for $p \geq 2$ and $n=4$}

We notice that, from [14], we know that if the function $K$ satisfies (1.1) and (H2) then the critical points at infinity are $\left(y_{i_{1}}, \ldots, y_{i_{s}}\right)_{\infty}$ where the $y_{i}$ 's are critical points of $K$ such that the matrix $M\left(y_{i_{1}}, \ldots, y_{i_{s}}\right)$, defined by (1.3), is positive definite. Note that in our case, the function $K$ can be flat (assumption (1.2)) near some critical points. Furthermore, Corollary 3.9 implies that there are other critical points at infinity which are of the type $(y)_{\infty}$ for $y \in I_{2}$. The aim of this subsection is to characterize the critical points at infinity in $V(p, \varepsilon)$ for $p \geq 2$. For this fact, we need to construct a pseudogradient on this set.

Proposition 3.10. Let $n=4$. Assume that $K$ satisfies $(\mathrm{H} 1, \mathrm{H} 2)$ and let $\beta:=$ $\max \{\beta(y): y$ satisfies (1.2)\}. For any $p \geq 2$, there exists a pseudogradient $W$ so that the following holds:

There is a positive constant $c>0$ independent of $u=\sum_{i \leq p} \alpha_{i} \delta_{\left(a_{i}, \lambda_{i}\right)} \in$ $V(p, \varepsilon)$ such that

(i) $(-\nabla J(u), W) \geq c \sum_{i \leq p} \frac{\left|\nabla K\left(a_{i}\right)\right|}{\lambda_{i}}+\frac{c}{\lambda_{i}^{\beta}}+c \sum_{k \neq r} \varepsilon_{k r}$

(ii) $\left(-\nabla J(u+\bar{v}), W+\frac{\partial \bar{v}}{\partial\left(\alpha_{i}, a_{i}, \lambda_{i}\right)}(W)\right) \geq c \sum_{i \leq p} \frac{\left|\nabla K\left(a_{i}\right)\right|}{\lambda_{i}}+\frac{c}{\lambda_{i}^{\beta}}+c \sum_{k \neq r} \varepsilon_{k r}$

(iii) $|W|$ is bounded. Furthermore, the only cases where the maximum of the $\lambda_{i}$ 's is not bounded is when the concentration points $\left(a_{1}, \cdots, a_{p}\right)$ satisfy: each point $a_{j}$ is close to a critical point $y_{i_{j}}$ of $K$ satisfying (1.1) with $i_{j} \neq i_{k}$ for $j \neq k$ and $\varrho\left(y_{i_{1}}, \ldots, y_{i_{p}}\right)>0$, where $\varrho\left(y_{i_{1}}, \ldots, y_{i_{p}}\right)$ denotes the least eigenvalue of $M\left(y_{i_{1}}, \ldots, y_{i_{p}}\right)$.

As a consequence of the above proposition and Corollary 3.9 we derive

Corollary 3.11. Let $n=4$. The only critical points at infinity are

- $\left(y_{i_{1}}, \ldots, y_{i_{q}}\right)_{\infty}$ such that the matrix $M\left(y_{i_{1}}, \ldots, y_{i_{q}}\right)$, defined by (1.3), is positive definite, where the $y_{i_{j}}$ 's are critical points of $K$ satisfying (1.1) with $i_{j} \neq i_{k}$ for $j \neq k$.

Such a critical point at Infinity has a Morse index equal to

$$
5 q-1-\sum_{j=1}^{q} \operatorname{Morse}\left(K, y_{i_{j}}\right)
$$


- $(y)_{\infty}$ where $y$ is a critical point of $K$ which belongs to $I_{2}$.

Such a critical point at Infinity has a Morse index equal to $4-\operatorname{index}(K, y)$ where index $(K, y):=\#\left\{b_{i} ; b_{i}<0\right\}$.

Proof of Proposition 3.10. Without loss of generality, we can assume that $\lambda_{1} \leq$ $\cdots \leq \lambda_{p}$. Let $M$ be a large positive constant and define

$$
\begin{aligned}
& I:=\{1\} \cup\left\{i \leq p: \lambda_{k} \leq M \lambda_{k-1} \forall k \leq i\right\}, \\
& I^{\prime}:=\left\{i \in I: a_{i} \text { is close to a critical point } y_{k_{i}} \text { satisfying (1.2) }\right\} .
\end{aligned}
$$

Note that the set $I$ contains the indices $i$ such that $\lambda_{i}$ and $\lambda_{1}$ are of the same order. We divide the set $V(p, \varepsilon)$ into four subsets:

$$
\begin{aligned}
F_{1} & :=\left\{\sum \alpha_{i} \delta_{\left(a_{i}, \lambda_{i}\right)}: \exists i \in I \text { such that } \lambda_{i}\left|\nabla K\left(a_{i}\right)\right| \geq C^{\prime}\right\}, \\
F_{2} & :=\left\{\sum \alpha_{i} \delta_{\left(a_{i}, \lambda_{i}\right)}: \forall i \in I, \lambda_{i}\left|\nabla K\left(a_{i}\right)\right| \leq 2 C^{\prime} \text { and } I^{\prime}=\emptyset\right\}, \\
F_{3} & :=\left\{\sum \alpha_{i} \delta_{\left(a_{i}, \lambda_{i}\right)}: I=I^{\prime}=\{1\} \text { and } \lambda_{1}\left|\nabla K\left(a_{1}\right)\right| \leq 2 C^{\prime}\right\}, \\
F_{4} & :=\left\{\sum \alpha_{i} \delta_{\left(a_{i}, \lambda_{i}\right)}: \forall i \in I, \lambda_{i}\left|\nabla K\left(a_{i}\right)\right| \leq 2 C^{\prime}, \#(I) \geq 2 \text { and } I^{\prime} \neq \emptyset\right\},
\end{aligned}
$$

where $C^{\prime}$ is a large positive constant. In each subset we will define a pseudogradient and the vector-field $W$ required in the proposition will be defined as a convex combination of all the pseudogradients. Let us denote by

$$
X_{i}:=\frac{1}{\lambda_{i}} \frac{\partial \delta_{i}}{\partial a_{i}} \frac{\nabla K\left(a_{i}\right)}{\left|\nabla K\left(a_{i}\right)\right|} \psi_{3}\left(\lambda_{i}\left|\nabla K\left(a_{i}\right)\right|\right) \quad \text { and } \quad Z_{i}:=\lambda_{i} \frac{\partial \delta_{i}}{\partial \lambda_{i}},
$$

where $\psi_{3}$ is a cut-off function defined by $\psi_{3}(t)=0$ if $t \leq \eta$ and $\psi_{3}(t)=1$ if $t \geq 2 \eta$ with $\eta$ a large positive constant $\left(2 \eta \leq C^{\prime}\right)$.

First, we remark that, by using Proposition 5.1 and (3.14), for each $q \leq p$, since $\eta$ is large, it is easy to obtain

$$
\begin{aligned}
& \left(-\nabla J(u), X_{i}\right) \geq c \psi_{3}\left(\lambda_{i}\left|\nabla K\left(a_{i}\right)\right|\right)\left(\frac{\left|\nabla K\left(a_{i}\right)\right|}{\lambda_{i}}+\frac{1}{\lambda_{i}^{2}}+O\left(\sum \varepsilon_{k i}\right)\right), \\
& \left(-\nabla J(u),-\sum_{i \geq q} 2^{i} Z_{i}\right) \geq c \sum_{k \geq q ; r \neq k} \varepsilon_{k r}+O\left(\sum_{i \geq q} \frac{1}{\lambda_{i}^{2}}\right)+o\left(\sum_{k \neq r} \varepsilon_{k r}\right) .
\end{aligned}
$$

- In $F_{1}$, we define $W_{1}:=\sum_{i \leq p} X_{i}-C_{1} \sum_{i \leq p} 2^{i} Z_{i}$, where $C_{1}$ is a large positive constant $\left(C_{1} \leq C^{\prime} / M^{p}\right)$. Using (3.32), (3.33) and the fact that there exists an index $i \in I$ such that $\lambda_{i}\left|\nabla K\left(a_{i}\right)\right| \geq C^{\prime}$, we derive the estimate of claim (i) in this case. 
- In $F_{2}$, we remark that we can write $u$ as

$$
u=u_{1}+u_{2} \text { where } u_{1}=\sum_{i \in I} \alpha_{i} \delta_{i} \text { and } u_{2}=\sum_{i \notin I} \alpha_{i} \delta_{i} .
$$

Observe that $u_{1} \in V(\#(I), \varepsilon)$. Since $I^{\prime}=\emptyset$ we can apply the vector field defined in [14] in this set, we will denote it by $Y\left(u_{1}\right)$. Hence we define $W_{2}:=Y\left(u_{1}\right)-$ $M \sum_{i \notin I} 2^{i} Z_{i}+\sum_{i \notin I} X_{i}$, where $M$ is defined in (3.29). Using [14] and (3.32), (3.33), we obtain

$$
\left(-\nabla J(u), W_{2}\right) \geq c \sum_{i \leq p} \frac{\left|\nabla K\left(a_{i}\right)\right|}{\lambda_{i}}+\frac{c}{\lambda_{i}^{2}}+c \sum_{k \neq r} \varepsilon_{k r} .
$$

Thus the estimate of claim (i) follows in this case.

- In $F_{3}$, we have $I=I^{\prime}=\{1\}$. Let $y_{k_{1}}$ be the critical point which is close to $a_{1}$. As in the proof of Proposition 3.5, we define $W_{3}^{1}$ and $W_{3}^{2}$ by (3.21) and (3.22) respectively with $n=4$. Following the proof of (3.25), we obtain

$$
\left(-\nabla J(u), W_{3}^{1}+W_{3}^{2}\right) \geq c \frac{\left|\nabla K\left(a_{1}\right)\right|}{\lambda_{1}}+\frac{c}{\lambda_{1}^{\beta}}+O\left(\sum \varepsilon_{k r}\right) .
$$

In this region, the pseudogradient will be defined as $W_{3}:=W_{3}^{1}+W_{3}^{2}-C \sum_{i>2} 2^{i} Z_{i}+$ $\sum_{i \leq p} X_{i}$ where $C$ is a large positive constant. Using (3.32), (3.33), (3.34) and the fact that $\lambda_{i}^{-2}=o\left(\varepsilon_{i j}\right)$ for each $i \notin I$ and $j \in I$, we derive

$$
\left(-\nabla J(u), W_{3}\right) \geq c \sum_{k \neq r} \varepsilon_{k r}+c \sum_{i \leq p} \frac{\left|\nabla K\left(a_{i}\right)\right|}{\lambda_{i}}+c \sum_{i \geq 2} \frac{c}{\lambda_{i}^{2}}+\frac{c}{\lambda_{1}^{\beta}} .
$$

Thus the estimate of claim (i) follows in this case.

- In $F_{4}$, we decrease all the variables $\lambda_{i}$ with different speeds. First, observe that $\lambda_{i}^{-2} \leq c_{M} \varepsilon_{i j}$ for each $i, j \in I, i \neq j$ and for $i \in I^{\prime}$ we have $\Delta K\left(a_{i}\right)=o(1)$. Thus, we define $W_{4}:=-\sum_{i \in I^{\prime}} Z_{i}-m^{\prime} \sum_{i \in I \backslash I^{\prime}} Z_{i}-\sum_{i \notin I} 2^{i} Z_{i}+m^{\prime \prime} \sum_{i \leq p} X_{i}$, where $m^{\prime}$ and $m^{\prime \prime}$ are small positive constants (they satisfy $m^{\prime} c_{M}$ and $m^{\prime \prime} / m^{\prime}$ are small). Using Proposition 5.1, (3.32), (3.33) and the fact that $\lambda_{i}^{-2}=o\left(\varepsilon_{i j}\right)$ for each $i \notin I$ and $j \in I$, we derive

$$
\left(-\nabla J(u), W_{4}\right) \geq c \sum_{k \neq r} \varepsilon_{k r}+c \sum_{i \leq p} \frac{\left|\nabla K\left(a_{i}\right)\right|}{\lambda_{i}}+\frac{c}{\lambda_{i}^{2}},
$$

which implies the estimate of claim (i) in this case.

Finally, the pseudogradient $W$ will be defined by a convex combination of $W_{1}$, $\ldots, W_{4}$. This vector field satisfies claim (i). 
Regarding claim (ii), as in [10] and [14], it follows from claim (i) and the estimate of $\|\bar{v}\|^{2}$ which is small with respect the lower-bound of claim (i).

Concerning claim (iii), it is easy to get that $|W|$ is bounded. Furthermore, from the definition of the $W_{i}$ 's, we remark that the maximum of the $\lambda_{i}$ 's is a decreasing function on the sets $F_{1}, F_{3}$ and $F_{4}$. However, in $F_{2}$, if $I \neq\{1, \ldots, p\}$, the maximum of the $\lambda_{i}$ 's is a decreasing function. But if $I=\{1, \ldots, p\}$, we have the same case as [14]. Thus claim (iii) follows. This completes the proof of the proposition.

\section{Proofs of the theorems}

Proof of Theorems 1.1 and 1.3. Before giving the proofs of our results, we will prove an abstract argument which provides a lower bound on the number of critical points of $J$. For that purpose let $\mathcal{K}_{\infty}$ be the set of critical points at infinity of $J$ and let $L_{0}$ be their maximal Morse index. We set:

$$
X:=\overline{\mathrm{U}_{\infty} \in \mathcal{K}_{\infty} W_{u}\left(z_{\infty}\right)},
$$

where $W_{u}\left(z_{\infty}\right)$ is the unstable manifold of the critical point at infinity $z_{\infty}$.

By a theorem of A. Bahri and P.H. Rabinowitz [13], we have that:

$$
X=\cup_{z \in \mathcal{K}_{\infty}} W_{u}(z) \cup \cup_{\left\{y \text { critical point dominated by a point } z_{\infty} \in \mathcal{K}_{\infty}\right\}} W_{u}(y) .
$$

Recall that $z_{\infty}$ is said to be dominated by another critical point at infinity $z_{\infty}^{\prime}$ if

$$
W_{u}\left(z_{\infty}^{\prime}\right) \cap W_{s}\left(z_{\infty}\right) \neq \varnothing .
$$

Therefore $X$ is a stratified set of top dimension $L_{0}$. Moreover it is contractible in $\Sigma^{+}$, by taking its suspension, that is, for $a \in \Sigma^{+}$, we consider $C(X):=\{t x+(1-$ t) $a ; x \in X\}$. Let $U$ such a contraction which is a stratified set of top dimension $L_{0}+1$. $U$ can also be deformed using the flow of $-\nabla J$. For dimension's reason the stable manifold of any critical point of Morse index $\geq L_{0}+2$ can be avoided during such a deformation see e.g. [34]. Therefore $U$ is deformed onto some set

$$
Z:=\cup_{z \in X} W_{u}(z) \cup \cup_{\{x \text { dominated by } X\}} W_{u}(x)
$$

where $z$ is a critical point or critical point at infinity.

We prove now the following proposition:

Proposition 4.1. Assuming that $K$ satisfies the condition $(\mathrm{H} 1)$ if $n=3$ and $(\mathrm{H} 1, \mathrm{H} 2)$ if $n=4$ and that all the critical points of $J$ having their Morse index less or equal to $L_{0}+1$ are nondegenerate, then it holds:

$$
\#\left\{\text { critical points of Morse index } \leq L_{0}+1\right\} \geq\left|1-\sum_{\tau \in \mathcal{K}_{\infty}}(-1)^{\iota(\tau)}\right|,
$$

where $\iota(\tau)$ denotes the Morse index of the critical point at Infinity $\tau$. 
Proof. Since $X$ is contractible in $Z$, we have from the exact sequence in homology that:

$$
\cdots \rightarrow H_{k}(X) \rightarrow H_{k}(Z) \rightarrow H_{k}(Z, X) \rightarrow H_{k-1}(X) \rightarrow H_{k-1}(Z) \rightarrow \cdots,
$$

where $H_{k}(X):=H_{k}(X, \mathbb{Q})$ is the $k$-th homology group with rational coefficients. Therefore it follows that

$$
\sum_{j=0}^{L_{0}+1}(-1)^{j}\left(\operatorname{dim} H_{j}(Z, X)+\operatorname{dim} H_{j}(X)\right)=1,
$$

which implies then

$$
\sum_{j=0}^{L_{0}+1} \operatorname{dim} H_{j}(Z, X) \geq\left|1-\sum_{j=0}^{L_{0}}(-1)^{j} \operatorname{dim} H_{j}(X)\right| .
$$

Now observe that the pair $(Z, X)$ is built by adding to $X$ the unstable manifold of other critical points of index $\leq L_{0}+1$. Namely each time we add one of these unstable manifolds, starting from $X$ and going with increasing index. At each step the new object we obtain has a total dimension of homology increased at most by one. Therefore the total homology of $(Z, X)$ has its dimension upper bounded by the number of critical points of index $\leq L_{0}+1$, not dominated by $X$. Therefore we have the following Lemma:

Lemma 4.2. Under the assumption that all the critical points with Morse index less or equal to $L_{0}+1$ are nondegenerate, it holds:

$$
\sum_{j=0}^{L_{0}+1} \operatorname{dim} H_{j}(Z, X) \leq \#\left\{\text { critical points of index } \leq L_{0}+1\right\} .
$$

The critical points in the above estimate are those in $Z$ but not in X.

Now Proposition 4.1 follows from formula (4.2) and Lemma 4.2. Indeed it follows from (4.2) that:

$$
\left|1-\sum_{j=0}^{L_{0}}(-1)^{j} \operatorname{dim} H_{j}(X)\right| \leq \sum_{j=0}^{L_{0}+1} \operatorname{dim} H_{j}(Z, X) .
$$

Now observe that:

$$
\sum_{j=0}^{L_{0}}(-1)^{j} \operatorname{dim} H_{j}(X)=\chi(X)(\text { The Euler Characteristic of } X) .
$$

Since by Proposition 7.24 of [13],

$$
X=\cup_{z_{\infty}} W_{u}\left(z_{\infty}\right) \cup \cup_{\left\{y \text { dominated by } z_{\infty} \in \mathcal{K}_{\infty}\right\}} W_{u}(y),
$$


it follows then that $\chi(X)$ is equal to

$$
\chi(X)=\sum_{z_{\infty} \in \mathcal{K}_{\infty}}(-1)^{\operatorname{index}\left(z_{\infty}\right)}+\sum_{y \in X, \text { critical point }}(-1)^{\operatorname{morse}(y)} .
$$

Therefore

$$
\left|1-\sum_{z_{\infty} \in \mathcal{K}_{\infty}}(-1)^{\operatorname{index}\left(z_{\infty}\right)}\right| \leq \sum_{j=0}^{L_{0}+1} \operatorname{dim} H_{j}(Z, X)+\#\{\text { critical point in } X\} .
$$

Using now the upperbound of Lemma 4.2 on the homology on the right hand side of the above formula, our claim in Proposition 4.1 follows.

Once Proposition 4.1 is proved, Theorems 1.1 and 1.3 follow immediately.

\section{Appendix}

This Appendix is devoted to some useful expansions of the gradient of $J$ near a potential critical points at infinity consisting of $p$ masses. Those propositions are extracted from [9] and [10] (with some change). In the sequel, we will write $\delta_{i}$ instead of $\delta_{\left(a_{i}, \lambda_{i}\right)}$.

Proposition 5.1. Let $n=3,4$ and $K$ be a $C^{2}$ positive function. For each $u=$ $\sum_{i=1}^{p} \alpha_{i} \delta_{i} \in V(p, \varepsilon)$, we have the following expansion

(a) $\left(\nabla J(u), \lambda_{i} \frac{\partial \delta_{i}}{\partial \lambda_{i}}\right)=-c_{2} J(u) \sum_{j \neq i} \alpha_{j} \lambda_{i} \frac{\partial \varepsilon_{i j}}{\partial \lambda_{i}}+2 J(u)^{\frac{2(n-1)}{n-2}} \alpha_{i}^{\frac{n+2}{n-2}} c_{1} \frac{\Delta K\left(a_{i}\right)}{\lambda_{i}^{2}}+R_{i}$, where $R_{i}=o\left(\sum_{k \neq r} \varepsilon_{k r}+\frac{1}{\lambda_{i}^{2}}\right)$.

(b) $\left(\nabla J(u), \frac{1}{\lambda_{i}} \frac{\partial \delta_{i}}{\partial a_{i}}\right)=-c_{4} J(u)^{\frac{2(n-1)}{n-2}} \alpha_{i}^{\frac{n+2}{n-2}} \frac{\nabla K\left(a_{i}\right)}{\lambda_{i}}+O\left(\sum_{k \neq i} \varepsilon_{k i}+\frac{1}{\lambda_{i}^{2}}\right)$.

(c) If $a_{i}$ is close to a critical point $y$ of $K$ satisfying (1.2), then the above estimate can be improved and we obtain

$$
\begin{aligned}
\left(\nabla J(u), \frac{1}{\lambda_{i}} \frac{\partial \delta_{i}}{\partial\left(a_{i}\right)_{k}}\right)= & -2 J(u)^{\frac{2(n-1)}{n-2}} \alpha_{i}^{\frac{n+2}{n-2}} \frac{1}{\lambda_{i}^{\beta}} \int_{\mathbb{R}^{n}} \frac{b_{k}\left|x_{k}+\lambda_{i}\left(a_{i}\right)_{k}\right|^{\beta} x_{k}}{\left(1+|x|^{2}\right)^{n+1}} d x \\
& +o\left(\frac{1}{\lambda_{i}^{\beta}}\right)+O\left(\sum_{k \neq r} \varepsilon_{k r}\right),
\end{aligned}
$$

where $k \leq n$ and $\left(a_{i}\right)_{k}$ is the $k$-th component of $a_{i}$ in some geodesic normal coordinates system. 
Furthermore, if we assume that $\lambda_{i}\left|a_{i}\right| \leq \rho$, where $\rho$ is a small positive constant, then

$$
\left(\nabla J(u), \lambda_{i} \frac{\partial \delta_{i}}{\partial \lambda_{i}}\right)=-c_{2} J(u) \sum_{j \neq i} \alpha_{j} \lambda_{i} \frac{\partial \varepsilon_{i j}}{\partial \lambda_{i}}+c \frac{\sum b_{j}}{\lambda_{i}^{\beta}}+o\left(\sum_{k \neq r} \varepsilon_{k r}+\frac{1}{\lambda_{i}^{\beta}}\right) .
$$

Proof. Claims (a) and (b) follow from [9]. Regarding claim (c), for simplicity, we assume that the critical point is zero. Following [9], it remains to expand the two integrals

$$
\begin{array}{rl}
\int_{\mathbb{S}^{n}} & K(x) \delta_{i}^{\frac{n+2}{n-2}} \frac{1}{\lambda_{i}} \frac{\partial \delta_{i}}{\partial\left(a_{i}\right)_{k}} \\
& =(n-2) \int_{\mathbb{R}^{n}}\left(\sum b_{j}\left|x_{j}\right|^{\beta}+R(x)\right) \frac{\lambda_{i}^{n+1}\left(x_{k}-\left(a_{i}\right)_{k}\right)}{\left(1+\lambda_{i}^{2}\left|x-a_{i}\right|^{2}\right)^{n+1}} d x \\
& =\frac{1}{\lambda_{i}^{\beta}} \int_{\mathbb{R}^{n}} b_{k}\left|x_{k}+\lambda_{i}\left(a_{i}\right)_{k}\right|^{\beta} \frac{x_{k}}{\left(1+|x|^{2}\right)^{n+1}} d x+o\left(\frac{1}{\lambda_{i}^{\beta}}\right) . \\
\int_{\mathbb{S}^{n}} & K(x) \delta_{i}^{(n+2) /(n-2)} \lambda_{i} \frac{\partial \delta_{i}}{\partial \lambda_{i}} \\
& =\frac{n-2}{2} \int_{\mathbb{R}^{n}} \sum b_{j}\left|x_{j}\right|^{\beta} \lambda_{i}^{n} \frac{1-\lambda_{i}^{2}\left|x-a_{i}\right|^{2}}{\left(1+\lambda_{i}^{2}\left|x-a_{i}\right|^{2}\right)^{n+1}} d x+o\left(\frac{1}{\lambda_{i}^{\beta}}\right) \\
& =\frac{n-2}{2} \frac{1}{\lambda_{i}^{\beta}} \sum b_{j} \int_{\mathbb{R}^{n}}\left|x_{j}+\lambda_{i}\left(a_{i}\right)_{j}\right|^{\beta} \frac{1-|x|^{2}}{\left(1+|x|^{2}\right)^{n+1}} d x+o\left(\frac{1}{\lambda_{i}^{\beta}}\right) .
\end{array}
$$

Observe that if $\lambda_{i}\left|a_{i}\right|$ is very small, it is easy to prove that

$$
\int_{\mathbb{R}^{n}}\left|x_{j}+\lambda_{i}\left(a_{i}\right)_{j}\right|^{\beta} \frac{1-|x|^{2}}{\left(1+|x|^{2}\right)^{n+1}} d x=\int_{\mathbb{R}^{n}}\left|x_{j}\right|^{\beta} \frac{1-|x|^{2}}{\left(1+|x|^{2}\right)^{n+1}} d x+o(1)<0,
$$

and the second integral in the above estimate is independent of $j$. Hence the result follows.

Lemma 5.2. For each $\eta \in \mathbb{R}$ and $i=1, \cdots, n$, we have

$$
\int_{\mathbb{R}^{n}}\left|x_{i}+\eta\right|^{\beta} \frac{x_{i}}{\left(1+|x|^{2}\right)^{n+1}} d x=0 \quad \text { iff } \quad \eta=0 .
$$

Furthermore, for each $\varepsilon>0$, there exists a positive constant $\bar{c}>0$ such that

$$
\left|\int_{\mathbb{R}^{n}}\right| x_{i}+\left.\eta\right|^{\beta} \frac{x_{i}}{\left(1+|x|^{2}\right)^{n+1}} d x \mid \geq \bar{c} \text { for each }|\eta| \geq \varepsilon .
$$




\section{References}

[1] S. Agmon, A. Douglis and L. NiREnBerg, Estimates near the boundary for solutions of elliptic partial differential equatins satisfying general boundary value conditions, I, Comm. Pure Appl. Math. 12 (1959), 623-727.

[2] A. Ambrosetti, J. Garcia Azorero and A. Peral, Perturbation of $-\Delta u+u^{\frac{(N+2)}{(N-2)}}=$ 0 , the Scalar Curvature Problem in $\mathbb{R}^{N}$ and related topics, J. Funct. Anal. 165 (1999), $117-149$.

[3] T. Aubin, Equations différentielles non linéaires et problème de Yamabe concernant la courbure scalaire, J. Math. Pures Appl. 55 (1976), 269-296.

[4] T. Aubin, Meilleures constantes de Sobolev et un théorème de Fredholm non linéaire pour la transformation conforme de la courbure scalaire, J. Funct. Anal. 32 (1979), 148-174.

[5] T. Aubin, "Some nonlinear problems in Riemannian geometry", Springer Monographs Math., Springer Verlag, Berlin, 1998.

[6] T. AUBIN and A. BAHRI, Méthodes de topologie algébrique pour le problème de la courbure scalaire prescrite. (French) [Methods of algebraic topology for the problem of prescribed scalar curvature], J. Math. Pures Appl. 76 (1997), 525-849.

[7] T. AUBIN and A. BAHRI, Une hypothèse topologique pour le problème de la courbure scalaire prescrite. (French) [A topological hypothesis for the problem of prescribed scalar curvature], J. Math. Pures Appl. 76 (1997), 843-850.

[8] T. Aubin and E. Hebey, Courbure scalaire prescrite, Bull. Sci. Math. 115 (1991), 125132.

[9] A. BAHRI, "Critical points at infinity in some variational problems", Pitman Res. Notes Math. Ser. Longman Sci. Tech. Harlow, Vol. 182, 1989.

[10] A. BAHRI, An invariant for Yamabe-type flows with applications to scalar curvature problems in high dimension, A celebration of J. F. Nash Jr., Duke Math. J. 81 (1996), 323-466.

[11] A. BAHRI and J. M. Coron, On a nonlinear elliptic equation involving the critical Sobolev exponent: the effect of topology of the domain, Comm. Pure Appl. Math. 41 (1988), 255294.

[12] A. BAHRI and J. M. CORON, The scalar curvature problem on the standard three dimensional spheres, J. Funct. Anal. 95 (1991), 106-172.

[13] A. BAhri and P. H. RaBinowitz, Periodic solutions of 3-body problems, Ann. Inst. H. Poincaré Anal. Non linéaire. 8 (1991), 561-649.

[14] M. Ben Ayed, Y. Chen, H. Chtioui and M. Hammami, On the prescribed scalar curvature problem on 4-manifolds, Duke Math. J. 84 (1996), 633-677.

[15] Ben Ayed, H. Chtioui and M. Hammami, The scalar curvature problem on higher dimensional spheres, Duke Math. J. 93 (1998), 379-424.

[16] H. BREzIS and J. M. Coron, Convergence of solutions of $H$-systems or how to blow bubbles, Arch. Ration. Mech. Anal. 89 (1985), 21-56.

[17] L. Caffarelli, B. Gidas and J. SprucK, Asymptotic symetry and local behavior of semilinear elliptic equations with critical Sobolev growth, Comm. Pure Appl. Math. 42 (1989), 271-297.

[18] S. A. Chang, M. J. Gursky and P. YAng, The scalar curvature equation on 2 and 3 spheres, Calc. Var. Partial Differential Equations 1 (1993), 205-229.

[19] K. C. CHANG and J. Q. LIU, On Nirenberg's problem, Internat. J. Math. (1993), 53-58.

[20] S. A. CHANG and P. YANG, Prescribing Gaussian curvature on $S^{2}$, Acta Math. 159 (1987), 215-259.

[21] S. A. CHANG and P. YANG, A perturbation result in prescribing scalar curvature on $S^{n}$, Duke Math. J. 64 (1991), 27-69.

[22] C. C. CHEN and C. S. LIN, Estimates of the scalar curvature via the method of moving planes I, Comm. Pure Appl. Math. 50 (1997), 971-1017. 
[23] C. C. CHEN and C. S. Lin, Estimates of the scalar curvature via the method of moving planes II, J. Differential Geom. 49 (1998), 115-178.

[24] C. C. CHEN and C. S. LIN, Prescribing the scalar curvature on $S^{n}, I$. A priori estimates, J. Differential Geom. 57 (2001), 67-171.

[25] H. Chtioui and M. Ould Ahmedou, Conformal metrics of prescribed scalar curvature on 4-manifolds: The degree zero case, Preprint 2008.

[26] A. DoLD, "Lectures on algebraic topology", Springer Verlag, Berlin, 1995.

[27] J. ESCOBAR and R. SCHOEN, Conformal metrics with prescribed scalar curvature, Invent. Math. 86 (1986), 243-254.

[28] E. HeBEy, Changements de metriques conformes sur la sphere, le problème de Nirenberg, Bull. Sci. Math. 114 (1990), 215-242.

[29] E. HEBEY, The isometry concentration method in the case of a nonlinear problem with Sobolev critical exponent on compact manifolds with boundary, Bull. Sci. Math. 116 (1992), $35-51$.

[30] Y. Y. LI, Prescribing scalar curvature on $S^{n}$ and related topics, Part I, J. Differential Equations, 120 (1995), 319-410.

[31] Y.Y. LI, Prescribing scalar curvature on $S^{n}$ and related topics, Part II : existence and compactness, Comm. Pure Appl. Math. 49 (1996), 437-477.

[32] C. S. LIN, Estimates of the scalar curvature via the method of moving planes III, Comm. Pure Appl. Math. 53 (2000), 611-646.

[33] P. L. LIONS, The concentration compactness principle in the calculus of variations. The limt case, Rev. Mat. Iberoamericana 1 (1985), I:165-201, II: 45-121.

[34] J. MILnOR, "Lectures on $h$-cobordism", Princeton University Press, Princeton, N.J., 1965.

[35] $\mathrm{O}$. REY, The role of Green's function in a nonlinear elliptic equation involving the critical Sobolev exponent, J. Funct. Anal. 89 (1990), 1-52.

[36] M. Schneider, Prescribing scalar curvature on $S^{3}$, Ann. Inst. H. Poincaré Anal. Non Linéaire 24 (2007), 563-587.

[37] R. Schoen and D. ZhANG, Prescribed scalar curvature on the n-sphere, Calc. Var. Partial Differential Equations 4 (1996), 1-25.

[38] M. STRUwe, A flow approach to Nirenberg problem, Duke Math. J. 128 (2005), 19-64.

[39] M. STRUwE, "Variational methods: Applications to nonlinear PDE \& Hamilton systems", Springer-Verlag, Berlin, 1990.

[40] M. STRUWE, A global compactness result for elliptic boundary value problems involving nonlinearities, Math. Z. 187 (1984), 511-517.

Département de Mathématiques

Faculté des Sciences de Sfax

Route Soukra, Sfax, Tunisia

mobenayed@yahoo.fr

Mathematisches institut

Universität Tübingen

Auf der Morgenstelle 10

D-72076 Tubingen, Germany

ahmedou@analysis.mathematik.uni-tuebingen.de 